Article

\title{
Enhanced Mechanical Properties of Multiscale Carbon Fiber/Epoxy Unidirectional Composites with Different Dimensional Carbon Nanofillers
}

\author{
Yu Liu ${ }^{1}{ }^{1}$, Dong-Dong Zhang ${ }^{2}$, Guang-Yuan Cui ${ }^{1}$, Rui-Ying Luo ${ }^{1, *}$ and Dong-Lin Zhao ${ }^{2, *}$ \\ 1 Research Institute for Frontier Science, Beihang University, Beijing 100029, China; \\ liuyu9175@buaa.edu.cn (Y.L.); m15300178689_3@163.com (G.-Y.C.) \\ 2 State Key Laboratory of Chemical Resource Engineering, Key Laboratory of Carbon Fiber and \\ Functional Polymers, Ministry of Education, Beijing University of Chemical Technology, \\ Beijing 100029, China; zhangdd8888@126.com \\ * Correspondence: ryluo@buaa.edu.cn (R.-Y.L.); dlzhao@mail.buct.edu.cn (D.-L.Z.)
}

Received: 3 August 2020; Accepted: 25 August 2020; Published: 26 August 2020

\begin{abstract}
Ammonia modified graphene-carbon nanotubes/continuous carbon fiber reinforced epoxy unidirectional multiscale composites (AMGNS-MWCNT/CFEP) were prepared by adding ammonia modified graphene and carbon nanotubes to an epoxy matrix to reduce agglomeration of carbon nanofillers in the epoxy matrix and improve composites properties. Fourier transform infrared spectroscopy (FTIR), scanning electron microscope (SEM), and universal testing machines were used to characterize the properties of carbon nanofillers, AMGNS-MWCNT/epoxy nanocomposites, and AMGNS-MWCNT/CFEP unidirectional composites. When the AMGNS-MWCNT content was $1.0 \mathrm{wt} \%$, flexural strength, the flexural modulus and interlaminar shear strength of AMGNS-MWCNT/CFEP unidirectional composites reached the maximum value of $1520.3 \mathrm{MPa}$, $138.88 \mathrm{GPa}$, and $87.80 \mathrm{MPa}$, respectively, which were $12.5 \%, 9.42 \%$, and $10.1 \%$ higher than that of carbon fiber reinforced epoxy unidirectional composites (CFEP). The synergistic mechanism of two carbon nanofillers in the matrix is discussed.
\end{abstract}

Keywords: graphene; carbon nanotubes; epoxy; mechanical property; composites

\section{Introduction}

Carbon fiber reinforced polymers (CFRPs) have excellent mechanical properties, low density, specific stiffness, and strength, and have important applications in aerospace, automobile, sports goods, and other industries [1-4]. Epoxy is a kind of thermosetting resin, which is widely used as the matrix of CFRPs. However, the inherent brittleness of epoxy limits its applications. In practical applications, nanomaterials are the most promising strengthening and toughening reinforcements for carbon fiber/epoxy composites. According to the different constraints in the space dimension, nanomaterials can be divided into zero-dimensional, one-dimensional, and two-dimensional nanomaterials. The nanomaterials in different dimensions have anisotropy to the load transfer direction [5]. Nanomaterials modified epoxy composites generally contain only one kind of nanomaterial of a single dimension [6-8]. However, the properties of the epoxy composites reinforced by single dimensional nanofillers will be improved while causing other properties to degrade [9-12]. To solve this problem, two carbon nanofillers with different dimensions are designed to be added to the epoxy matrix to improve the comprehensive performance of CFRPs $[13,14]$.

The surface and edge of graphene oxide prepared by the Hummers method contain a large number of oxygen-containing functional groups [15-17]. The oxygen-containing functional groups provide the possibility of introducing modified substances on the surface of graphene. Therefore, modification of 
graphene can be achieved by modifying and reducing the graphene oxide [18,19]. Graphene prepared by the graphene oxide reduction method, which is widely used in the laboratory, often has certain structural defects, and the carbon atoms located in defects have strong chemical activity, so it is also possible to modify graphene at this active site. The covalent modification of graphene mainly involves four reactions: nucleophilic substitution, electrophilic addition, condensation, and addition polymerization [20-29]. The lone electron pair in ammonia reacts with the oxygen-containing groups on the surface and edges of graphene, mainly by nucleophilic substitution. One is to produce Brønsted acid by $\mathrm{NH}_{4}{ }^{+}$, and the other is to produce Lewis acid in the form of $-\mathrm{NH}_{2}$ [30].

In this study, ammonia modified graphene and carbon nanotubes were prepared and used as reinforcements to form carbon fiber/epoxy unidirectional multiscale composites. The combination of graphene nanosheets and carbon nanotubes can effectively prevent the agglomeration and winding by taking advantage of their differences in the direction of force transfer in spatial dimensions. The mechanical properties of the composites were improved, and the synergistic mechanism of ammonia modified graphene and carbon nanotubes was studied.

\section{Materials and Methods}

\subsection{Preparation of Ammonia Modified Graphene-Carbon Nanotubes/Continuous Carbon Fiber Reinforced Epoxy Composites}

Graphene nanosheets (GNS) were prepared by the chemical oxidation-rapid thermal expansion reduction-ultrasonic stripping method [31]. A total of $0.5 \mathrm{~g}$ graphite oxide was quickly put into a preheated muffle furnace at $1050{ }^{\circ} \mathrm{C}$ and kept for $30 \mathrm{~s}$ to obtain expanded graphite. After sieving, the expanded graphite was dissolved into anhydrous ethanol at a ratio of $0.2 \mathrm{~g} / 100 \mathrm{~mL}$, and ultrasonic treatment was performed for $15 \mathrm{~h}$. After the ethanol was volatilized, the sample was dried in a vacuum oven at $120^{\circ} \mathrm{C}$ for $4 \mathrm{~h}$ to obtain GNS. Ammonia modified graphene nanosheets (AMGNS) were prepared by mixing $90 \mathrm{~mL}$ hydrogen peroxide and $150 \mathrm{~mL}$ ammonia with $1.5 \mathrm{~g}$ GNS. In the preparation process, the mixture was ultrasonically treated for $4 \mathrm{~h}$. After filtration, the filter cake was washed to neutral with deionized water, and then dried at $65^{\circ} \mathrm{C}$ inside the vacuum oven for $12 \mathrm{~h}$.

A certain amount of carbon nanotubes (CNT) were refluxed with $100 \mathrm{~mL}$ concentrated nitric acid for $5 \mathrm{~h}$. After filtration, the filter cake was washed to neutral with deionized water, and then dried at $60{ }^{\circ} \mathrm{C}$ inside the vacuum oven for $24 \mathrm{~h}$.

Equally proportional AMGNS and modified multi-wall carbon nanotubes (MWCNT) $(0.1 \mathrm{wt} \%$, $0.5 \mathrm{wt} \%, 1.0 \mathrm{wt} \%, 2.0 \mathrm{wt} \%$, and $3.0 \mathrm{wt} \%$ ) were added into the resin solution. The mass ratio of epoxy (E-51, Sinopec Baling Petrochemical Co., Hunan, China) to curing agent (MeTHPA, Beijing Chemical Reagent Co., Beijing, China) was 10:7. After mechanical stirring and ultrasonic reaction for $6 \mathrm{~h}$, a small amount of promoters (DMP-30, Beijing Chemical Reagent Co., Beijing, China) were added, and the ultrasonic reaction continued for $0.5 \mathrm{~h}$. Then, the reaction solution was placed in a vacuum oven at $50{ }^{\circ} \mathrm{C}$ to debubble for $0.5 \mathrm{~h}$. The mixed solution was poured into a stainless-steel mold and cured in sections with temperature rise. AMGNS-MWCNT/epoxy nanocomposites were obtained after demolding. The neat epoxy and AMGNS-MWCNT/epoxy nanocomposites of different components are named as EP, CG0.1, CG0.5, CG1.0, CG2.0, and CG3.0.

The process was repeated and the mixture was poured into a stainless-steel mold with a copper wire frame wound with T300 (Toray Industries Co., Tokyo, Japan, 3K) continuous carbon fiber. AMGNS-MWCNT/continuous carbon fiber reinforced epoxy unidirectional composites (AMGNS-MWCNT/CFEP) was obtained by curing, stripping, and processing procedures. The volume fraction of carbon fiber was $60 \mathrm{vol} \%$. The contents of AMGNS-MWCNT were $0 \mathrm{wt} \%, 0.1 \mathrm{wt} \%, 0.5 \mathrm{wt} \%$, $1.0 \mathrm{wt} \%$, and $2.0 \mathrm{wt} \%$ with an equally proportion. The neat continuous carbon fiber reinforced epoxy unidirectional composite and AMGNS-MWCNT/CFEP unidirectional composites of different components are named as CFEP, CGFP0.1, CGFP0.5, CGFP1.0, and CGFP2.0. 


\subsection{Characterization}

The surface functional groups of AMGNS-MWCNT were investigated by Fourier transform infrared spectroscopy (FT-IR, Nicolet, Nexus670, Madison, WI, USA). The surface morphology of composites was investigated by scanning electron microscope (SEM, Hitachi, S-4700, Tokyo, Japan). The tensile, flexural, and interlaminar shear properties of composites were tested by universal testing machines (Instron, Instron ${ }^{\mathrm{TM}} 1185$ and Instron ${ }^{\mathrm{TM}}$ 5567, Instron Corporation, Canton, MA, USA).

The tensile strength was calculated using Equation (1):

$$
\sigma_{t}=\frac{P}{b h}
$$

where $\sigma_{t}$ is the tensile strength (MPa); $P$ is the maximum load $(\mathrm{N}) ; b$ is the specimen width $(\mathrm{mm})$; and $h$ is the specimen thickness (mm).

The tensile modulus was calculated using Equation (2):

$$
E_{t}=\frac{L_{0} \Delta P}{b h \Delta L}
$$

where $E_{t}$ is the tensile modulus (MPa); $L_{0}$ is the gauge length (mm); $\Delta P$ is the load increment $(\mathrm{N}) ; \Delta L$ is the gauge length increment corresponding to $\triangle P(\mathrm{~mm})$.

Elongation at break was calculated using Equation (3):

$$
\varepsilon_{t}=\frac{\Delta L_{b}}{L_{0}} \times 100 \%
$$

where $\varepsilon_{t}$ is the elongation at break (\%); and $\Delta L_{b}$ is the elongation at break within the gauge length $L_{0}$ of the sample (mm).

The flexural strength was calculated using Equation (4):

$$
\sigma_{f}=\frac{3 P L}{2 b h^{2}}
$$

where $\sigma_{f}$ is the flexural strength (MPa) and $L$ is the span $(\mathrm{mm})$.

The flexural strain was calculated using Equation (5):

$$
\varepsilon_{f}=\frac{6 s h}{L^{2}} \times 100 \%
$$

where $\varepsilon_{f}$ is the flexural strain (\%) and $s$ is the deflection ( $\left.\mathrm{mm}\right)$.

The flexural modulus was calculated using Equation (6):

$$
E_{f}=\frac{L^{3} \Delta P}{4 b h^{3} \Delta S}
$$

where $E_{f}$ is the flexural modulus (MPa) and $\Delta S$ is the deflection increment corresponding to $\Delta P(\mathrm{~mm})$.

The interlaminar shear strength was calculated using Equation (7):

$$
\tau_{s}=\frac{3 P_{b}}{4 b h}
$$

where $\tau_{s}$ is the interlaminar shear strength (MPa) and $P_{b}$ is the maximum load $(\mathrm{N})$. 


\section{Results and Discussion}

\subsection{The Properties of AMGNS and Modified CNT}

To improve the bonding performance of GNS and CNT to the epoxy matrix, the surface modification of these two carbon nanofillers was carried out. The edge of GNS prepared by the thermal reduction of graphite oxide contained many carboxyl functional groups. These active groups can react with ammonia to obtain AMGNS. The FTIR spectrum of GNS before and after ammonia modification are shown in Figure 1a. In the ammonia modified GNS spectrum, the peaks appeared at $1560 \mathrm{~cm}^{-1}\left(-\mathrm{NH}_{2}\right.$ stretching), $1400 \mathrm{~cm}^{-1}$ (C-N stretching) [32,33], and blue shift (shift to lower wavenumbers) of all characteristic peaks, indicating that ammonia had successfully modified graphene. FTIR spectrum of CNT treated by concentrated nitric acid refluxes are shown in Figure $1 \mathrm{~b}$. The peaks appearing at $1732 \mathrm{~cm}^{-1}(C=\mathrm{O}$ stretching) indicate that the surface of the CNT had been modified by oxygen-containing functional groups [1].

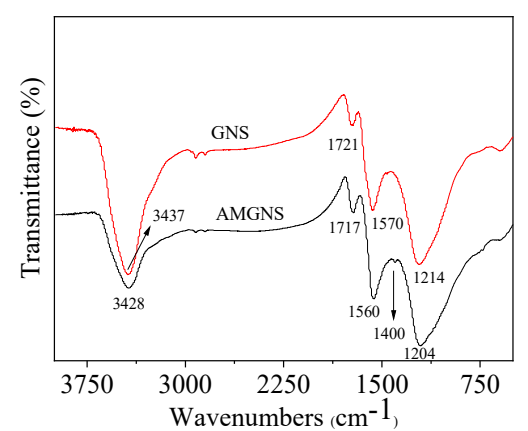

(a)

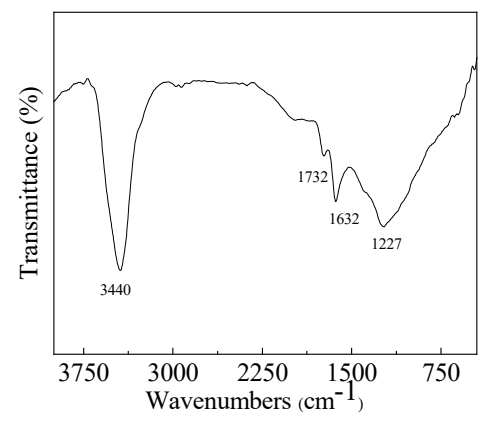

(b)

Figure 1. FTIR spectrum of (a) GNS and AMGNS; (b) CNT after concentrated nitric acid treatment.

\subsection{Mechanical Properties of AMGNS-MWCNT/Epoxy Nanocomposites}

The tensile strength, tensile modulus, and elongation at break of AMGNS-MWCNT/epoxy nanocomposites are shown in Table 1 and Figure 2. With the increase in the content of carbon nanofillers, the tensile strength and elongation at break of the composites increased first and then decreased. When the content of AMGNS-MWCNT was $2.0 \mathrm{wt} \%$, the tensile strength and elongation at break of the AMGNS-MWCNT/epoxy nanocomposites reached the maximum value of 63.53 $\mathrm{MPa}$ and 3.53\%, respectively. Compared with neat epoxy, the tensile strength and elongation at break of AMGNS-MWCNT/epoxy nanocomposites increased by $20.7 \%$ and $34.7 \%$, respectively. When the content of AMGNS-MWCNT was $0.1 \mathrm{wt} \%$, the tensile strength and elongation at break of AMGNS-MWCNT/epoxy nanocomposites were not significantly different from that of neat epoxy. This is because the content of AMGNS-MWCNT in the resin matrix was too low, there was no good contact between the nanofillers, and they were not evenly dispersed in the matrix, which tended to form defects in the materials. Therefore, the nanocomposites are more likely to fracture when subjected to external forces. When the content of nanofillers increased to a certain extent $(3 \mathrm{wt} \%)$, the agglomeration of AMGNS or MWCNT became more serious, and their dispersion in the epoxy matrix became worse, resulting in more internal defects, which reflected poor mechanical properties. The tensile modulus of the nanocomposites increased with the content of AMGNS-MWCNT. When the content of AMGNS-MWCNT was $3.0 \mathrm{wt} \%$, the tensile modulus of the AMGNS-MWCNT/epoxy nanocomposites reached the maximum value of $3.07 \mathrm{GPa}$, which was $24.8 \%$ higher than that of neat epoxy. 


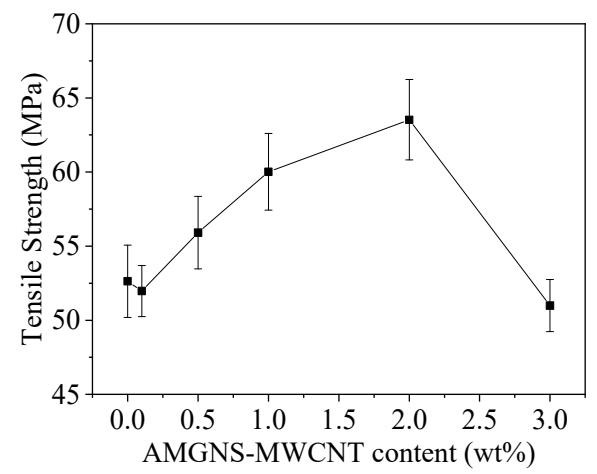

(a)

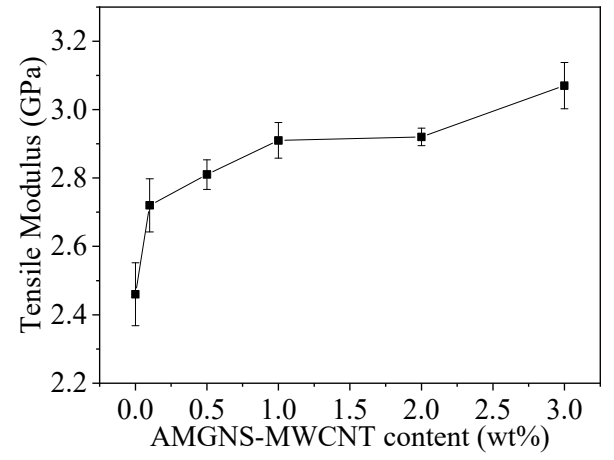

(b)

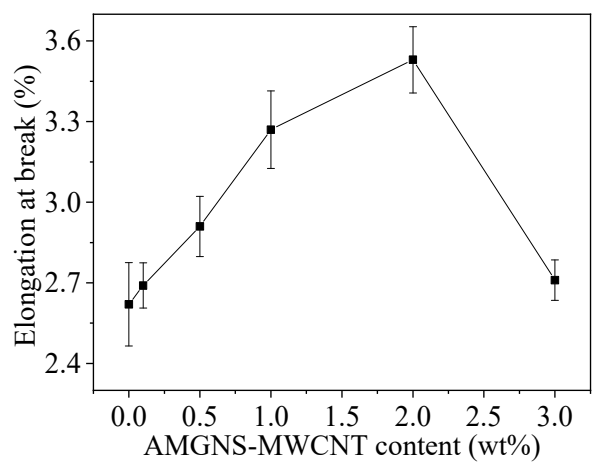

(c)

Figure 2. Tensile properties of the AMGNS-MWCNT/epoxy nanocomposites: (a) tensile strength; (b) tensile modulus; (c) elongation at break.

Table 1. Tensile properties of AMGNS-MWCNT/epoxy nanocomposites with different AMGNS-MWCNT content.

\begin{tabular}{cccc}
\hline Specimens & Tensile Strength/MPa & Tensile Modulus/GPa & Elongation at Break/\% \\
\hline EP & 52.63 & 2.46 & 2.62 \\
CG0.1 & 51.97 & 2.72 & 2.69 \\
CG0.5 & 55.91 & 2.81 & 2.91 \\
CG1.0 & 60.02 & 2.91 & 3.27 \\
CG2.0 & 63.53 & 2.92 & 3.53 \\
CG3.0 & 50.99 & 3.07 & 2.71 \\
\hline
\end{tabular}

SEM images of the fractured surface of AMGNS-MWCNT/epoxy nanocomposites are shown in Figure 3. When the content of AMGNS-MWCNT was low $(<0.5 \mathrm{wt} \%)$, the fractured surface was relatively smooth, and MWCNT was sporadically distributed in the epoxy matrix, which makes it difficult to strengthen the epoxy resin. When the content of AMGNS-MWCNT was high (>3.0 wt $\%)$, the agglomeration of MWCNT was very serious, and the GNS did not have a good coupling effect with CNT, which would easily lead to the deterioration of the mechanical properties of composites. When the content of AMGNS-MWCNT was within a certain range (1.0-2.0 wt\%), AMGNS-MWCNT were relatively dispersed in the matrix, and the agglomeration and entangling phenomenon were not obvious. This is because one-dimensional long-range ordered CNT can bridge the GNS, improve the specific surface area of the AMGNS-MWCNT filler, and reduce the degree of agglomeration, thus contributing to the improvement of the mechanical properties of the composite. 

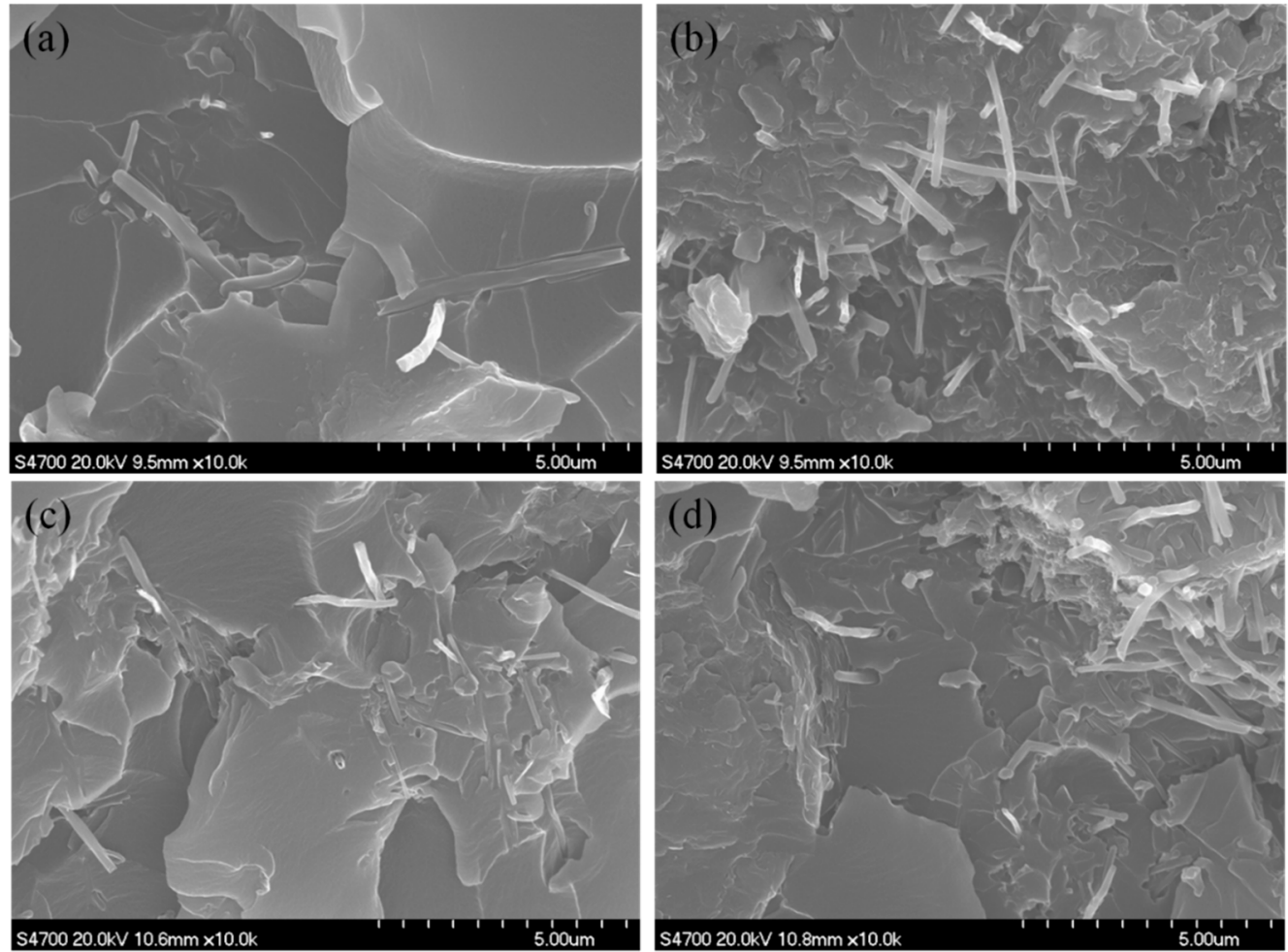

Figure 3. SEM micrographs of the fractured surface of the epoxy composites with AMGNS-MWCNT mixture contents of (a) $0.5 \mathrm{wt} \%$, (b) $1.0 \mathrm{wt} \%$, (c) $2.0 \mathrm{wt} \%$, and (d) $3.0 \mathrm{wt} \%$. (Supplementary Materials Figures S1 and S2).

The flexural strength, flexural modulus, and flexural strain of the AMGNS-MWCNT/epoxy nanocomposites are shown in Table 2 and Figure 4. With the increase in AMGNS-MWCNT content, the flexural strength, flexural modulus, and flexural strain of AMGNS-MWCNT/epoxy nanocomposites increased first and then decreased. When the content of AMGNS-MWCNT was $2.0 \mathrm{wt} \%$, the flexural strength and flexural strain of the AMGNS-MWCNT/epoxy nanocomposites reached a maximum of 108.47 MPa and 3.23\%, respectively. Compared with neat epoxy, the flexural strength and flexural strain of the AMGNS-MWCNT/epoxy nanocomposites increased by $55.5 \%$ and $11.8 \%$, respectively. When the content of AMGNS-MWCNT was $1.0 \mathrm{wt} \%$, the flexural modulus of the AMGNS-MWCNT/epoxy nanocomposites reached a maximum of $3.99 \mathrm{GPa}$. Compared with neat epoxy, the flexural modulus increased by $23.5 \%$.

Table 2. Flexural properties of the AMGNS-MWCNT/epoxy nanocomposites with different AMGNS-MWCNT content.

\begin{tabular}{cccc}
\hline Specimens & Flexural Strength/MPa & Flexural Modulus/GPa & Flexural Strain/\% \\
\hline EP & 69.75 & 3.23 & 2.89 \\
CG0.1 & 95.19 & 3.46 & 2.90 \\
CG0.5 & 99.58 & 3.58 & 2.96 \\
CG1.0 & 104.34 & 3.99 & 3.17 \\
CG2.0 & 108.47 & 3.78 & 3.23 \\
CG3.0 & 87.01 & 3.71 & 2.85 \\
\hline
\end{tabular}




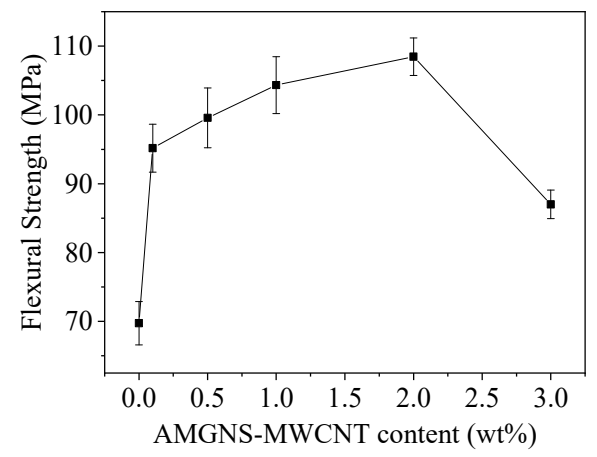

(a)

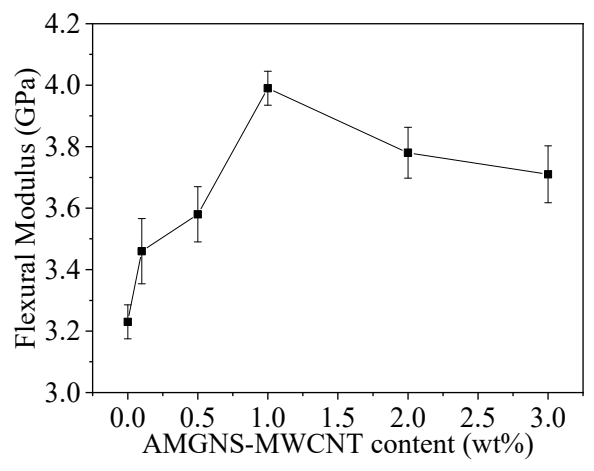

(b)

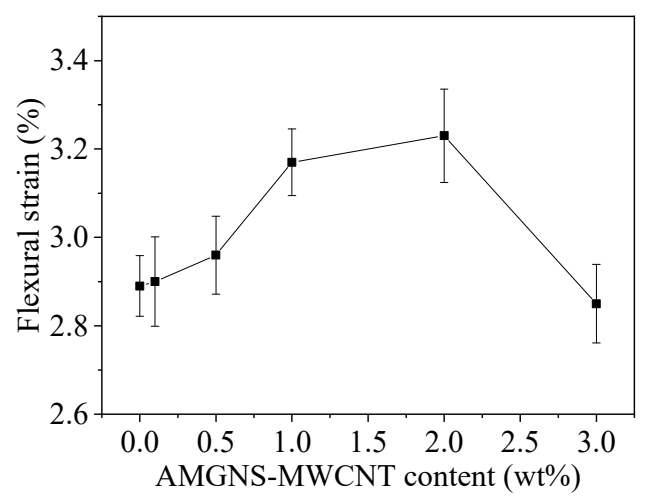

(c)

Figure 4. Flexural properties of the AMGNS-MWCNT/epoxy nanocomposites: (a) flexural strength; (b) flexural modulus; (c) flexural strain.

\subsection{Mechanical Properties of AMGNS-MWCNT/CFEP Unidirectional Composite}

The flexural properties and interlaminar shear strength for AMGNS-MWCNT/CFEP unidirectional composites are shown in Table 3. The flexural strength and flexural modulus of AMGNS-MWCNT/CFEP unidirectional composite with different AMGNS-MWCNT contents are shown in Figure 5. With the increase in the AMGNS-MWCNT content, the flexural strength and flexural modulus of the AMGNS-MWCNT/CFEP unidirectional composite increased first and then decreased. When the content of AMGNS-MWCNT was $1.0 \mathrm{wt} \%$, the flexural strength and flexural modulus of the AMGNS-MWCNT/CFEP unidirectional composite reached the maximum value of $1520.3 \mathrm{MPa}$ and 138.88 GPa, respectively. Compared with the CFEP composite without any fillers, the flexural strength and flexural modulus increased by $12.5 \%$ and $9.42 \%$, respectively.

Table 3. Flexural properties and interlaminar shear strength of the AMGNS-MWCNT/CFEP unidirectional composites with different AMGNS-MWCNT content.

\begin{tabular}{cccc}
\hline Specimens & Flexural Strength/MPa & Flexural Modulus/GPa & Interlaminar Shear Strength/MPa \\
\hline CFEP & 1351.7 & 126.92 & 79.81 \\
CGFP0.1 & 1402.6 & 132.69 & 81.28 \\
CGFP0.5 & 1480.9 & 135.52 & 83.63 \\
CGFP1.0 & 1520.3 & 138.88 & 87.80 \\
CGFP2.0 & 1428.8 & 131.54 & 82.17 \\
\hline
\end{tabular}




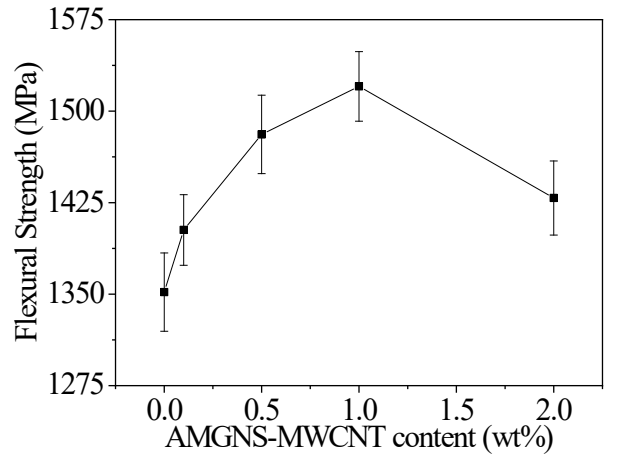

(a)

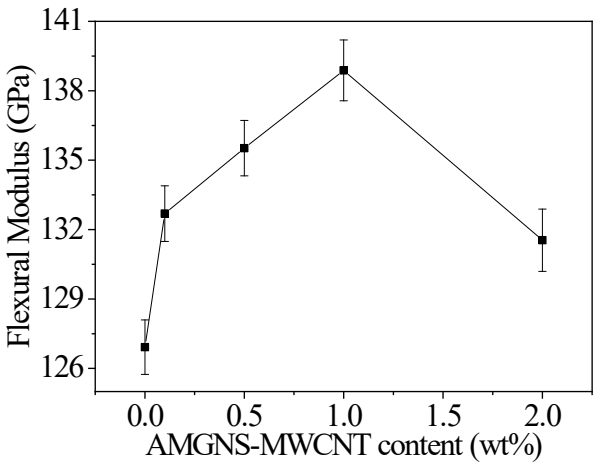

(b)

Figure 5. Flexural properties of the AMGNS-MWCNT/CFEP unidirectional composites: (a) flexural strength; (b) flexural modulus.

SEM images of the flexural fracture surface of the AMGNS-MWCNT/CFEP unidirectional composites with a content of $1.0 \mathrm{wt} \%$ of AMGNS-MWCNT are shown in Figure 6. The volume fraction of carbon fiber was $60 \mathrm{vol} \%$. The carbon fiber was evenly distributed in the composite, and there was no obvious crack around it. It can be seen from Figure $6 b$ that when the AMGNS-MWCNT/CFEP unidirectional composite was broken, the pull-out effect of carbon fibers was dominant. This is because the addition of one-dimensional CNT can prevent the agglomeration of GNS and reduce the formation of internal defects in the material. The AMGNS-MWCNT fillers form a good bridging effect between the carbon fibers and epoxy matrix. When an external force is applied, the synergy between the two nanofillers can effectively delay the crack propagation to carbon fibers, and the extraction of carbon fibers and the interaction of fillers consume a large amount of applied energy, which makes the composite have excellent mechanical properties.
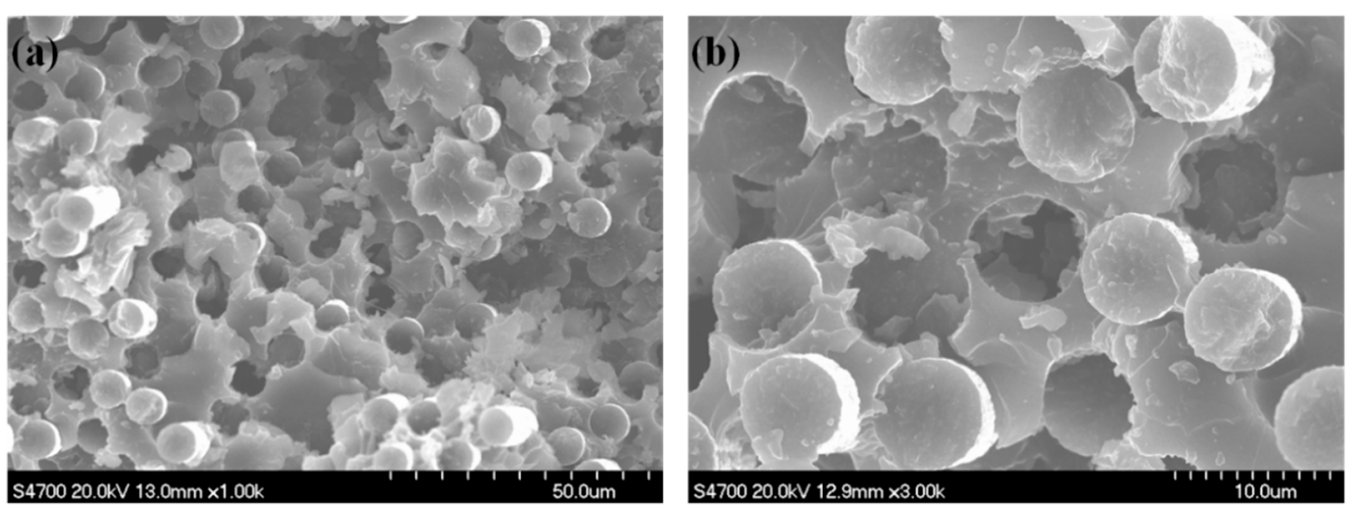

Figure 6. SEM images of the AMGNS-MWCNT/CFEP unidirectional composites: (a) low-magnification micrograph of the fiber and surrounding matrix; (b) high-magnification view of the fiber/matrix interface.

Interlaminar shear strength of the AMGNS-MWCNT/CFEP unidirectional composites with different AMGNS-MWCNT contents are shown in Figure 7. With the increase in AMGNS-MWCNT content, the interlaminar shear strength of AMGNS-MWCNT/CFEP unidirectional composites increased first and then decreased. When the content of AMGNS-MWCNT was $1.0 \mathrm{wt} \%$, the interlaminar shear strength of the AMGNS-MWCNT/CFEP unidirectional composite reached the maximum value of $87.80 \mathrm{MPa}$, which was $10.1 \%$ higher than that of the CFEP without any fillers. This indicates that the AMGNS-MWCNT/CFEP unidirectional composites had good interlaminar interfacial properties and the bonds between the fibers were tight. Compared with the traditional carbon fiber composite materials, the existence of the two-dimensional nanofillers not only exerts the synergistic effect between the fillers, 
but also has the dimensional effect between the two fillers. Due to the different directions of conduction when different dimensional materials face the stress, the AMGNS-MWCNT/CFEP unidirectional composites can rapidly and effectively transfer the stress to the three-dimensional direction under the action of the applied load. When these stresses meet the GNS and CNT, the formation of cracks will be slowed down. Therefore, the AMGNS-MWCNT/CFEP unidirectional composites have relatively high interlaminar shear strength.

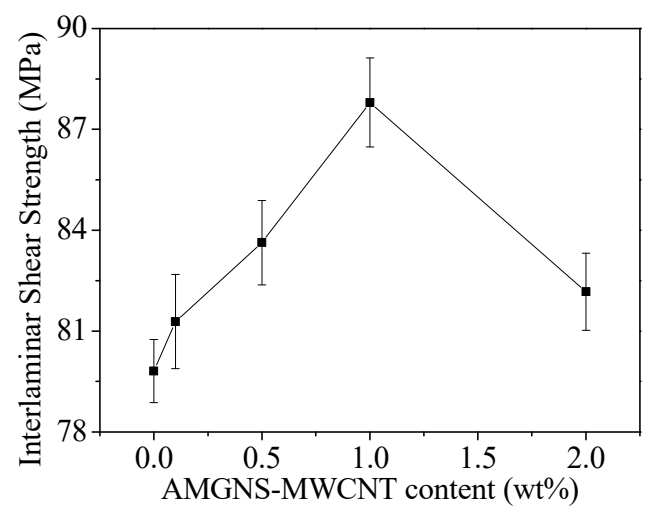

Figure 7. Interlaminar shear strength of the AMGNS-MWCNT/CFEP unidirectional composites.

The dispersion and compatibility of two-dimensional GNS and one-dimensional CNT in the epoxy matrix play an important role in enhancing the mechanical properties of composites. Figure 8 shows the simulation of the strengthening and toughening mechanism of two-dimensional GNS and one-dimensional CNT fillers in composites. Due to its large length-diameter ratio, CNTs are easy to be wound and agglomerated in the matrix, which limits their excellent performance. GNS are also prone to pile-up in the matrix due to their van der Waals' forces and $\pi-\pi$ bonding, which results in their inability to give full play to its performance as a load transfer in the composite material. When both fillers are used as reinforcing materials for the composites, the defects are less likely to form in the case that the two fillers are well dispersed. The fibrous CNTs effectively limit the agglomeration between GNS due to the $\pi-\pi$ bond. GNS and CNTs are combined to form a three-dimensional network structure, which improves the interface contact area between the reinforcement and the matrix and generates a strong molecular binding force. When subjected to external forces, the load can develop along the three-dimensional direction, thus improving the fracture resistance of the composite.

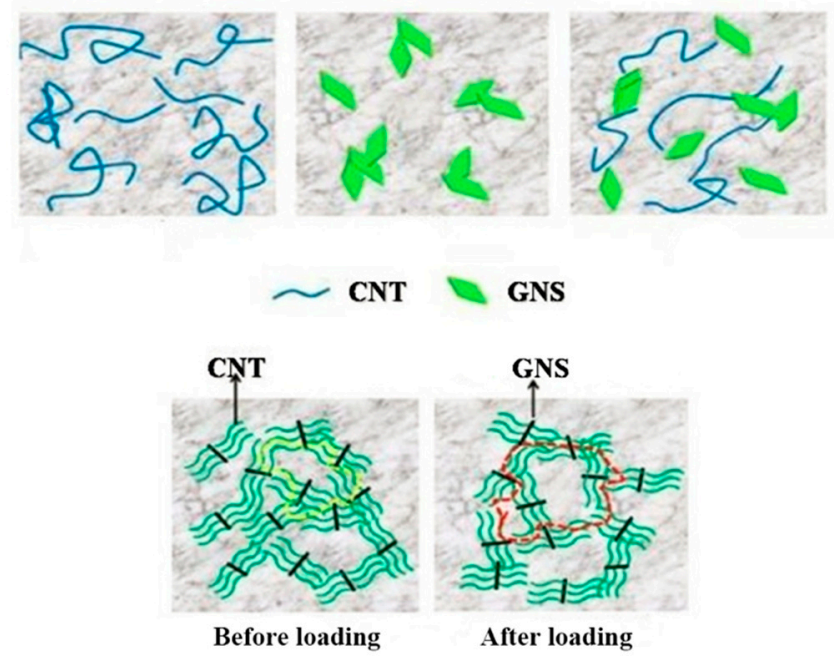

Figure 8. Schematic images of the reinforcement dispersion in the epoxy composites. 


\section{Conclusions}

This study demonstrates the improvement in the mechanical properties of multiscale carbon fiber/epoxy unidirectional composites with different dimensional carbon nanofillers. When the content of AMGNS-MWCNT was $2.0 \mathrm{wt} \%$ and $1.0 \mathrm{wt} \%$, the AMGNS-MWCNT/epoxy nanocomposites and AMGNS-MWCNT/CFEP unidirectional composites showed the best performance, respectively. The tensile strength, elongation at break, flexural strength, and flexural strain of the AMGNS-MWCNT/epoxy nanocomposites could reach to $63.53 \mathrm{MPa}, 3.53 \%, 108.47 \mathrm{MPa}$, and 3.23\%, respectively, which were $20.7 \%, 34.7 \%, 55.5 \%$, and $11.8 \%$ higher than that of neat epoxy. Furthermore, the flexural strength, flexural modulus, and interlaminar shear strength of the AMGNS-MWCNT/CFEP unidirectional composites reached the maximum value of $1520.3 \mathrm{MPa}, 138.88 \mathrm{GPa}$, and $87.80 \mathrm{MPa}$, respectively, which were $12.5 \%, 9.42 \%$, and $10.1 \%$ higher than that of CFEP. This is because two-dimensional GNS and one-dimensional CNT fillers are combined to form a three-dimensional network structure in composites to improve the interface contact area between the reinforcement and the matrix. The three-dimensional network structure can effectively disperse the load and improve the mechanical properties of the composites.

Supplementary Materials: The following are available online at http://www.mdpi.com/2079-4991/10/9/1670/s1, Figure S1: SEM micrographs of the epoxy composites with AMGNS-MWCNT mixture contents of $3.0 \mathrm{wt} \%$. (a) the fractured surface of the composites; (b) the agglomeration CNTs in matrix of the composites. Figure S2: SEM micrographs of the fractured surface of the epoxy composites with AMGNS-MWCNT mixture contents of (a) 1.0 $w t \%$, (b) $2.0 w t \%$.

Author Contributions: Conceptualization, Y.L., D.-D.Z., G.-Y.C., R.-Y.L., and D.-L.Z.; Methodology, Y.L. and D.-D.Z.; Software, D.-D.Z.; Validation, Y.L. and D.-D.Z.; Investigation, Y.L. and D.-D.Z.; Resources, R.-Y.L. and D.-L.Z.; Data curation, D.-D.Z.; Writing-original draft preparation, Y.L.; Writing-review and editing, R.-Y.L. and D.-L.Z.; Visualization, Y.L. and D.-D.Z.; Supervision, R.-Y.L. and D.-L.Z.; Project administration, R.-Y.L. and D.-L.Z. All authors have read and agreed to the published version of the manuscript.

Funding: This research received no external funding.

Conflicts of Interest: The authors declare no conflict of interest.

\section{References}

1. Park, S.J.; Park, S.J. Effect of ozone-treated single-walled carbon nanotubes on interfacial properties and fracture toughness of carbon fiber-reinforced epoxy composites. Compos. Part A Appl. Sci. Manuf. 2020, 105937. [CrossRef]

2. Yao, Z.; Wang, C.; Qin, J.; Su, S.; Wang, Y.; Wang, Q.; Yu, M.; Wei, H. Interfacial improvement of carbon fiber/epoxy composites using one-step method for grafting carbon nanotubes on the fibers at ultra-low temperatures. Carbon 2020, 164, 133-142. [CrossRef]

3. Cao, S.; Zhis, W.U.; Wang, X. Tensile Properties of CFRP and Hybrid FRP Composites at Elevated Temperatures. J. Compos. Mater. 2009, 43, 315-330.

4. Zhang, W.; Deng, X.; Sui, G.; Yang, X. Improving interfacial and mechanical properties of carbon nanotube-sized carbon fiber/epoxy composites. Carbon 2019, 145, 629-639. [CrossRef]

5. Gerasin, V.A.; Antipov, E.M.; Karbushev, V.V.; Kulichikhin, V.; Karpacheva, G.P.; Talroze, R.V.; Kudryavtsev, Y.V. New approaches to the development of hybrid nanocomposites: From structural materials to high-tech applications. Russ. Chem. Rev. 2013, 82, 303-332. [CrossRef]

6. Ávila, A.F.; Yoshida, M.I.; Carvalho, M.G.R.; Dias, E.C.; de Ávila Junior, J. An investigation on post-fire behavior of hybrid nanocomposites under bending loads. Compos. Part B Eng. 2010, 41, 380-387. [CrossRef]

7. Aldajah, S.; Haik, Y. Transverse strength enhancement of carbon fiber reinforced polymer composites by means of magnetically aligned carbon nanotubes. Mater. Des. 2012, 34, 379-383. [CrossRef]

8. Zhang, X.; Fan, X.; Yan, C.; Li, H.; Zhu, Y.; Li, X.; Yu, L. Interfacial microstructure and properties of carbon fiber composites modified with graphene oxide. ACS Appl. Mater. Interfaces 2012, 4, 1543-1552. [CrossRef]

9. Gibson, R.F. A review of recent research on mechanics of multifunctional composite materials and structures. Compos. Struct. 2010, 92, 2793-2810. [CrossRef] 
10. Uddin, M.F.; Sun, C.T. Strength of unidirectional glass/epoxy composite with silica nanoparticle-enhanced matrix. Compos. Sci. Technol. 2008, 68, 1637-1643. [CrossRef]

11. Liu, S.; Zhang, H.; Zhang, Z.; Zhang, T.; Sprenger, S. Tailoring the Mechanical Performance of Epoxy Resin by Various Nanoparticles. Polym. Polym. Compos. 2008, 16, 527-533. [CrossRef]

12. Manjunatha, C.M.; Taylor, A.C.; Kinloch, A.J.; Sprenger, S. The effect of rubber micro-particles and silica nano-particles on the tensile fatigue behaviour of a glass-fibre epoxy composite. J. Mater. Sci. 2009, 44, 342-345. [CrossRef]

13. Yang, S.Y.; Lin, W.N.; Huang, Y.L.; Tien, H.W.; Wang, J.Y.; Ma, C.C.; Li, S.M.; Wang, Y.S. Synergetic effects of graphene platelets and carbon nanotubes on the mechanical and thermal properties of epoxy composites. Carbon 2011, 49, 793-803. [CrossRef]

14. Li, W.; Dichiara, A.; Bai, J. Carbon nanotube-graphene nanoplatelet hybrids as high-performance multifunctional reinforcements in epoxy composites. Compos. Sci. Technol. 2013, 74, 221-227. [CrossRef]

15. Kuila, T.; Bose, S.; Mishra, A.K.; Khanra, P.; Kim, N.H.; Lee, J.H. Chemical functionalization of graphene and its applications. Prog. Mater. Sci. 2012, 57, 1061-1105. [CrossRef]

16. Bielawski, C.; Dreyer, D.R.; Park, S.; Ruoff, R.S. The chemistry of graphene oxide. Chem. Soc. Rev. 2010, 39, 228-240.

17. Bai, H.; Li, C.; Shi, G. Functional composite materials based on chemically converted graphene. Adv. Mater. 2011, 23, 1089-1115. [CrossRef]

18. Wang, X.; Xing, W.; Zhang, P.; Song, L.; Yang, H.; Hu, Y. Covalent functionalization of graphene with organosilane and its use as a reinforcement in epoxy composites. Compos. Sci. Technol. 2012, 72, 737-743. [CrossRef]

19. Fang, M.; Wang, K.; Lu, H.; Yang, Y.; Nutt, S. Single-layer graphene nanosheets with controlled grafting of polymer chains. J. Mater. Chem. 2010, 20, 1982-1992. [CrossRef]

20. Bourlinos, A.B.; Gournis, D.; Petridis, D.; Szabó, T.; Szeri, A.; Dékány, I. Graphite oxide: Chemical reduction to graphite and surface modification with primary aliphatic amines and amino acids. Langmuir 2003, 19, 6050-6055. [CrossRef]

21. Lomeda, J.R.; Doyle, C.D.; Kosynkin, D.V.; Hwang, W.F.; Tour, J.M. Diazonium functionalization of surfactant-wrapped chemically converted graphene sheets. J. Am. Chem. Soc. 2008, 130, 16201-16206. [CrossRef] [PubMed]

22. Zhu, Y.; Higginbotham, A.L.; Tour, J.M. Covalent functionalization of surfactant-wrapped graphene nanoribbons. Chem. Mater. 2009, 21, 5284-5291. [CrossRef]

23. Yang, H.; Shan, C.; Li, F.; Han, D.; Zhang, Q.; Niu, L. Covalent functionalization of polydisperse chemically-converted graphene sheets with amine-terminated ionic liquid. Chem. Commun. 2009, 26, 3880-3882. [CrossRef] [PubMed]

24. Park, S.; Dikin, D.A.; Nguyen, S.T.; Ruoff, R.S. Graphene oxide sheets chemically cross-linked by polyallylamine. J. Phys. Chem. C 2009, 113, 15801-15804. [CrossRef]

25. Avinash, M.B.; Subrahmanyam, K.S.; Sundarayya, Y.; Govindaraju, T. Covalent modification and exfoliation of graphene oxide using ferrocene. Nanoscale 2010, 2, 1762-1766. [CrossRef]

26. Fang, M.; Wang, K.; Lu, H.; Yang, Y.; Nutt, S. Covalent polymer functionalization of graphene nanosheets and mechanical properties of composites. J. Mater. Chem. 2009, 19, 7098-7105. [CrossRef]

27. Stankovich, S.; Piner, R.D.; Nguyen, S.T.; Ruoff, R.S. Synthesis and exfoliation of isocyanate-treated graphene oxide nanoplatelets. Carbon 2006, 44, 3342-3347. [CrossRef]

28. Georgakilas, V.; Bourlinos, A.B.; Zboril, R.; Steriotis, T.A.; Dallas, P.; Stubos, A.K.; Trapalis, C. Organic functionalisation of graphenes. Chem. Commun. 2010, 46, 1766-1768. [CrossRef]

29. Sundaram, R.S.; Gómez-Navarro, C.; Balasubramanian, K.; Burghard, M.; Kern, K. Electrochemical modification of graphene. Adv. Mater. 2008, 20, 3050-3053. [CrossRef]

30. Seredych, M.; Bandosz, T.J. Mechanism of ammonia retention on graphite oxides: Role of surface chemistry and structure. J. Phys. Chem. C 2007, 111, 15596-15604. [CrossRef]

31. Dhakate, S.R.; Chauhan, N.; Sharma, S.; Tawale, J.; Singh, S.; Sahare, P.D.; Mathur, R.B. An approach to produce single and double layer graphene from re-exfoliation of expanded graphite. Carbon 2011, 49, 1946-1954. [CrossRef] 
32. Jia, Y.; Yu, H.; Zhang, Y.; Dong, F.; Li, Z. Cellulose acetate nanofibers coated layer-by-layer with polyethylenimine and graphene oxide on a quartz crystal microbalance for use as a highly sensitive ammonia sensor. Colloids Surf. B Biointerfaces 2016, 148, 263-269. [CrossRef] [PubMed]

33. Barrera-Andrade, J.M.; Rojas-García, E.; García-Valdés, J.; Valenzuela, M.A.; Albiter, E. Incorporation of amide functional groups to graphene oxide during the photocatalytic degradation of free cyanide. Mater. Lett. 2020, 17, 128538. [CrossRef]

(C) 2020 by the authors. Licensee MDPI, Basel, Switzerland. This article is an open access article distributed under the terms and conditions of the Creative Commons Attribution (CC BY) license (http://creativecommons.org/licenses/by/4.0/). 